\title{
Central serous chorioretinopathy complicating systemic corticosteroid treatment
}

\author{
MASATO WAKAKURA AND SATOSHI ISHIKAWA \\ From the Department of Ophthalmology, Kitasato University, School of Medicine, 1-15-1 Kitasato, \\ Sagamihara, Kanagawa 228, Japan
}

SUMmARY Central serous chorioretinopathy developed in 2 cases of retrobulbar neuritis during systemic treatment with corticosteroids. Fluorescein angiography confirmed leakage surrounded by central serous detachment of the retina. In one case the central serous chorioretinopathy recurred 3 times during 3 separate courses of treatment. Spontaneous recovery accompanied a reduction in steroid treatment to a low level. It is suggested that high dosage corticosteroids may damge the posterior blood-ocular barrier.

Maculopathy complicating treatment with systemic corticosteroids (prednisolone, ${ }^{1}$ ) or corticotrophic hormone (depot tetracosactrin ${ }^{2}$ ) has been reported. We have previously noted worsening of central serous chorioretinopathy (CSR) in 5 cases during systemic corticosteroid therapy. In this report we describe the development of CSR during treatment of retrobulbar nêuritis (RBN) in 2 cases, one of which developed 3 separate attacks of CSR during 3 separate courses of corticosteroid therapy.

\section{Case reports}

\section{CASE 1}

A 39-year-old man was admitted to our hospital with acute bilateral RBN of unknown origin. His corrected vision was $0.4 \mathrm{OD}$ and $0.01 \mathrm{OS}$. Ophthalmoscopic examination was normal except for a few small subretinal yellowish dots in the right macular area. There was a past history of CSR in the right eye. Corticosteroid treatment with betamethasone $10 \mathrm{mg}$ per day in $500 \mathrm{ml}$ of $5 \%$ glucose was started. This was a high initial dosage but was thereafter gradually reduced. On the sixth day of therapy, when the steroid dosage was $8 \mathrm{mg}$ daily and the total dosage $52 \mathrm{mg}$, CSR with a detachment of about 2 disc diameters was noted in the right eye and rapidly increased to 5 disc diameters. Following termination of corticosteroid therapy the extent of the detachment reduced to about 2 disc diameters. Vision remained poor in each eye, and consequently a second course of betamethasone with a total dosage of $123 \mathrm{mg}$ over 18 days was started. During this second course there was an increase in the CSR, but the detachment completely disappeared about 20 days after the termination of corticosteroid therapy. At that time the visual acuity was $1.0 \mathrm{OD}$ and $0.05 \mathrm{OS}$. Because of continuing poor vision the patient requested a third course of corticosteroid therapy. Two months were allowed to elapse to allow adrenal function to recover.

Neither a right central scotoma nor dye leakage on fluorescein angiography was detected before the start of the third course (Fig. 1A). Betamethasone $16 \mathrm{mg}$ per day was given and the dosage gradually reduced. The total dosage was $141 \mathrm{mg}$ over 15 days. On the third day. of treatment vision in the right eye deteriorated to $0 \cdot 6$, and a central scotoma and CSR were noted. The same leakage site as before was confirmed by fluorescein angiography site (Fig. 1B). The leakage site was surrounded by a pigment epithelial detachment. On the eleventh day (Fig. 1C) the leakage changed to a mushroom or jet type with a retinal detachment of about 6 disc diameters. The CSR did not improve until the daily dosage of betamethasone was reduced to $2 \mathrm{mg}$. The detachment disappeared about 18 days after the termination of corticosteroid therapy with a vision of $1.0 \mathrm{OD}$ and $0 \cdot 1$ OS. No dye leakage was seen at this stage (Fig. 1D).

CASE 2

A 44-year-old man was admitted to our hospital with bilateral RBN of unknown origin. His corrected vision was 0.02 OD and 0.04 OS. Ophthalmoscopic exam- 

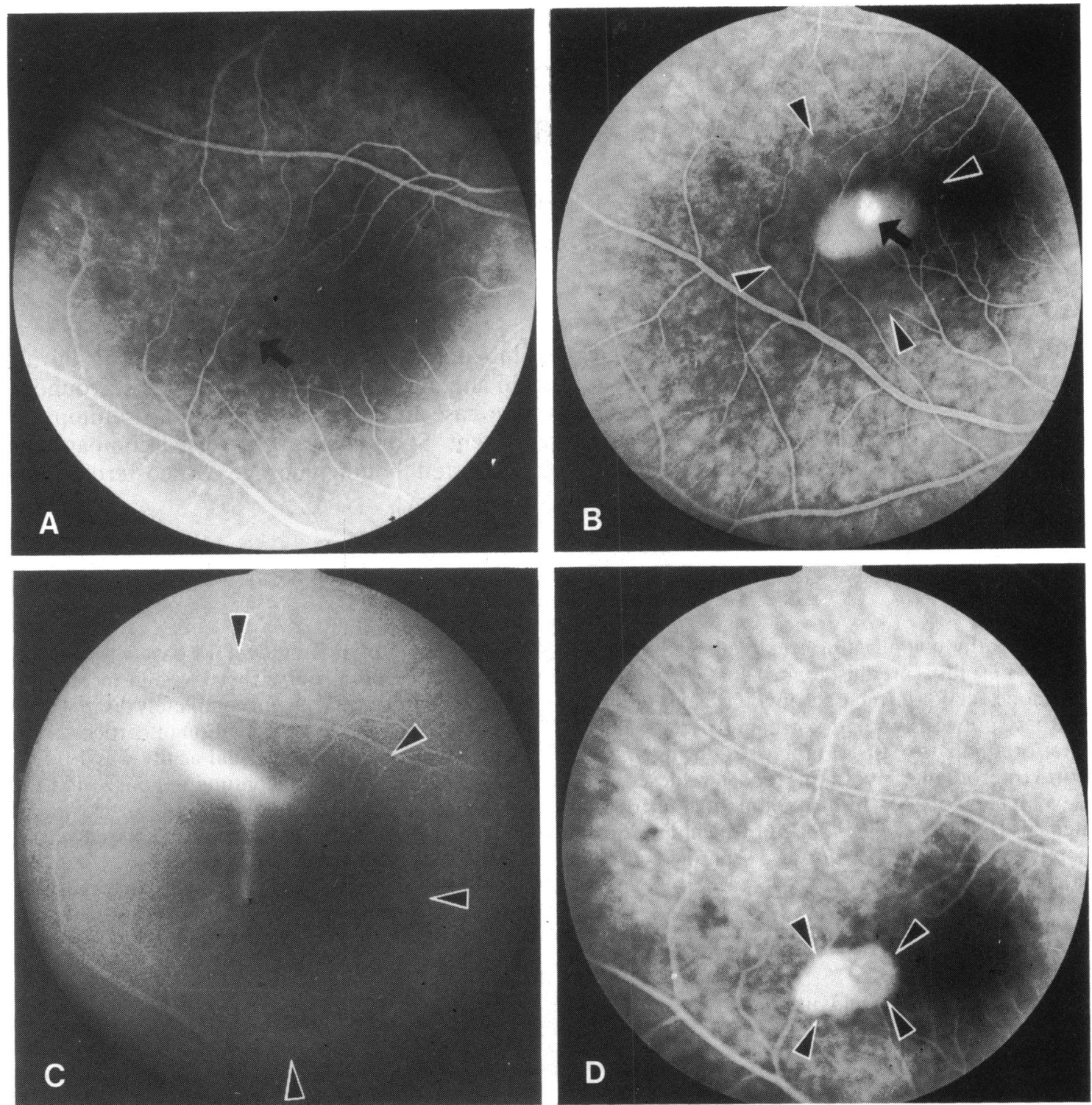

Fig. 1 Fluorescein angiograms before (A) and during (B-D) treatment with systemic corticosteroids in a patient who had developed CSR during 2 previous courses of steroid therapy for RBN. A Only small hyperfluorescent spots without dye leakage are seen at the earlier leakage site (arrow). B On the third day of betamethasone treatment. Note CSR of 2 disc diameters (triangles) with both dye leakage (arrow) and pigment epithelial detachment. $\mathrm{COn}$ the eleventh day of treatment. Area of CSR is enlarged to about 6 disc diameters (triangles) with dye leakage of mushroom or jet type. D On eighteenth day after termination of treatment. Neither retinal detachment nor dye leakage is seen. Triangles indicate the border of a pigment epithelial detachment.

ination was normal except for parafoveal depigmentation in the left eye. No retinal detachment was present. Granular hyperfluorescent lesions without dye leakage (window defects) were observed by fluorescein angiography, but the fovea was not involved (Fig. 2A). Treatment with betamethasone 10 $\mathrm{mg}$ per day in $500 \mathrm{ml}$ of $5 \%$ glucose was started. The dosage was gradually reduced. On the twelfth day after the start of treatment when the dose of betamethasone was $4 \mathrm{mg}$ per day CSR with a detachment of about 2 disc diameters was noted temporal to the left fovea. Leakage from 2 sites was confirmed by 

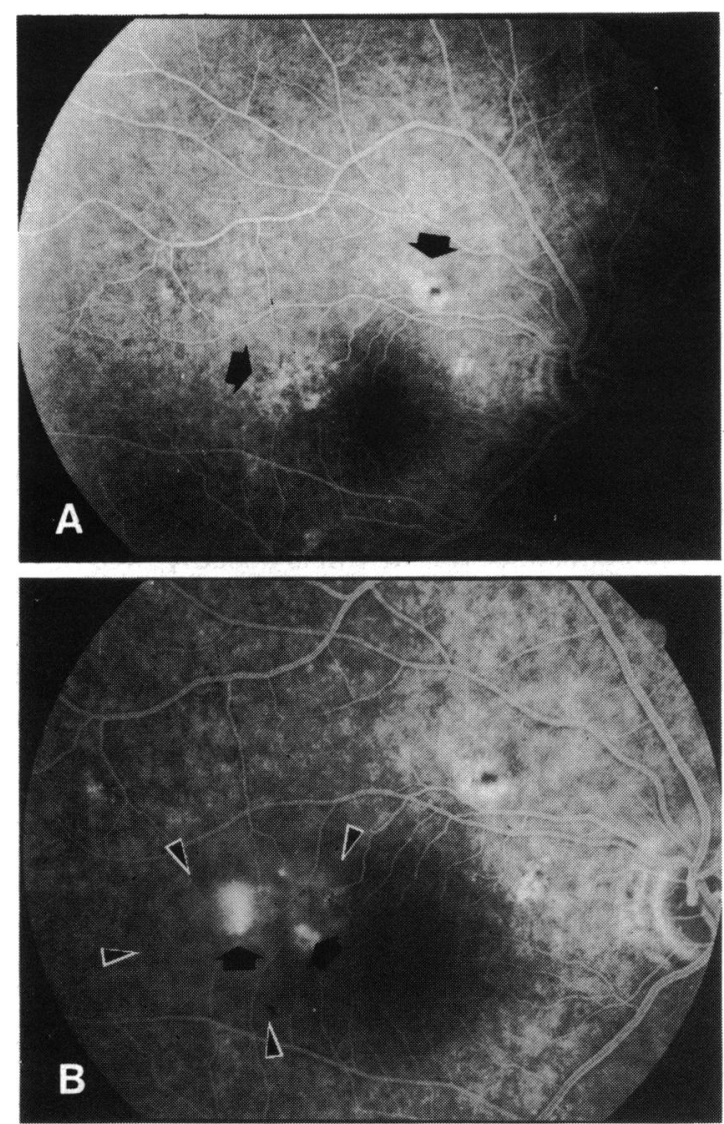

Fig. 2 Fluorescein angiogram before (A) and after (B) betamethasone treatment in case 2 . A Note granular hyperflüorescent lesions without dye leakage in the paramacular area (arrows). B On the twelfth day of betamethasone treatment. Note CSR with detachment of about 2 disc diameters (triangles) and 2 leakage sites (arrows).

fluorescein angiography (Fig. 2B). Enlargement of the detachment to about 3 disc diameters continued until daily dosage was reduced to $2 \mathrm{mg}$. The detachment disappeared about 35 days after termination of corticosteroid administration. The final visual acuity was $0.5 \mathrm{OD}$ and $0.5 \mathrm{OS}$.

\section{Discussion}

Although corticosteroid therapy has been widely used for the treatment of CSR, clinical studies ${ }^{3}$ have failed to show any benefit with regard to either final visual outcome or duration of the disorder. Gass ${ }^{4}$ has expressed the view that corticosteroid therapy has no place in the management of CSR, and the effects of systemic corticosteroids on experimentally induced $\mathrm{CSR}^{5}$ are unclear.

Apart from lack of benefit there is some evidence that systemic corticosteroids may actually be harmful to the posterior blood-ocular barrier. Maculopathy apparently induced by systemic steroid therapy has been reported, ${ }^{12}$ and we ourselves ${ }^{6}$ have noted worsening of CSR during corticosteroid therapy in 5 cases.

In the present 2 cases of $\mathrm{RBN}$ treated with systemic betamethasone damage to the posterior blood-ocular barrier was confirmed by fluorescein angiography. The appearances resembled idiopathic CSR and in both patients the CSR was unilateral. The possibility exists that the development of CSR during corticosteroid therapy was coincidental, but the recurrent attacks of CSR during 3 separate courses of steroid therapy suggest that the therapy was causally related to the CSR. Both patients had evidence of pre-existing pathology of the macula, and it may be that an anatomical weakness of the blood-ocular barrier such as a focal defect in the adherence of the retinal pigment epithelium to Bruch's membrane was associated with a local susceptibility to corticosteroid treatment.

A possible effect of endogenous corticosteroids on the posterior blood-ocular barrier is suggested in a case reported by Chumbley and Frank, ${ }^{7}$ where 4 attacks of CSR developed during 4 successive pregnancies, each attack resolving spontaneously after delivery or spontaneous abortion:

In the present cases CSR appeared to be associated with corticosteroid treatment in high dosage, and it is suggested that caution be exercised when corticosteroids in high dosage are considered for patients with a history of CSR or ophthalmoscopic evidence of previous CSR.

\section{References}

1 Jain IS, Singh' K. Maculopathy: a corticosteroid side effect. $J$ All-India Ophthalmol Soc 1966; 14: 250-2.

2 Williamson J, Nuki G. Macular lesions during systemic therapy with depot tetracosactrin. BrJ Ophthalmol 1970; 54: 405-9.

3 Nanjiani M. Long-term follow-up of central serous retinopathy. Trans Ophthalmol Soc UK 1977; 97: 656-61.

4 Gass JDM. Idiopathic central serous chorioretinopathy. In: Stereoscopic atlas of macular disease. 2nd ed. St Louis: Mosby, $1977 ; 28-40$.

5 Yoshioka H, Katsume Y, Akune H. Experimental central serous chorioretinopathy in monkey eyes: fluorescein angiographic findings. Ophthalmologica 1982; 185: 168-78.

6 Wakakura M, Ishikawa S. An evaluation of corticosteroid treatment for central serous chorioretinopathy. Rinsho Ganka 1980; 34: 123-9.

7 Chumbley LC, Frank RN. Central serous retinopathy and pregnancy. Am J Ophthalmol 1974; 77: 158-60. 